\title{
Impact of cancer and cardiovascular disease on in-hospital outcomes of COVID-19 patients: results from the american heart association COVID-19 cardiovascular disease registry
}

David M. Tehrani ${ }^{1}$, Xiaoyan Wang ${ }^{2}$, Asim M. Rafique ${ }^{1}$, Salim S. Hayek ${ }^{3}$, Joerg Herrmann ${ }^{4}$, Tomas G. Neilan ${ }^{5}$, Pooja Desai ${ }^{6}$, Alicia Morgans ${ }^{7}$, Juan Lopez-Mattei ${ }^{8}$, Rushi V. Parikh ${ }^{1}$ and Eric H. Yang ${ }^{1,9^{*}}$

\begin{abstract}
Background: While pre-existing cardiovascular disease (CVD) appears to be associated with poor outcomes in patients with Coronavirus Disease 2019 (COVID-19), data on patients with CVD and concomitant cancer is limited. The purpose of this study is to evaluate the effect of underlying CVD and CVD risk factors with cancer history on in-hospital mortality in those with COVID-19.

Methods: Data from symptomatic adults hospitalized with COVID-19 at 86 hospitals in the US enrolled in the American Heart Association's COVID-19 CVD Registry was analyzed. The primary exposure was cancer history. The primary outcome was in-hospital death. Multivariable logistic regression models were adjusted for demographics, CVD risk factors, and CVD. Interaction between history of cancer with concomitant CVD and CVD risk factors were tested.

Results: Among 8222 patients, 892 (10.8\%) had a history of cancer and 1501 (18.3\%) died. Cancer history had significant interaction with CVD risk factors of age, body mass index (BMI), and smoking history, but not underlying CVD itself. History of cancer was significantly associated with increased in-hospital death (among average age and $\mathrm{BMl}$ patients, adjusted odds ratio $[\mathrm{aOR}]=3.60,95 \%$ confidence interval $[\mathrm{Cl}]$ : $2.07-6.24 ; p<0.0001$ in those with a smoking history and $\mathrm{aOR}=1.33,95 \% \mathrm{Cl}: 1.01-1.76 ; p=0.04$ in non-smokers). Among the cancer subgroup, prior use of chemotherapy within 2 weeks of admission was associated with in-hospital death $(\mathrm{aOR}=1.72,95 \% \mathrm{Cl}$ : 1.05-2.80; $p=0.03)$. Underlying CVD demonstrated a numerical but statistically nonsignificant trend toward increased mortality ( $\mathrm{aOR}=1.18,95 \% \mathrm{Cl}: 0.99-1.41 ; p=0.07)$.

Conclusion: Among hospitalized COVID-19 patients, cancer history was a predictor of in-hospital mortality. Notably, among cancer patients, recent use of chemotherapy, but not underlying CVD itself, was associated with worse survival. These findings have important implications in cancer therapy considerations and vaccine distribution in cancer patients with and without underlying CVD and CVD risk factors.
\end{abstract}

Keywords: COVID-19, Cancer, Malignancy, Cardiovascular disease, Mortality, Severe disease, Thromboembolic disease

*Correspondence: Ehyang@mednet.ucla.edu

${ }^{1}$ Division of Cardiology, Department of Medicine, University of California, Los Angeles Health System, Los Angeles, CA, USA

Full list of author information is available at the end of the article

\section{Introduction}

The novel coronavirus Severe Respiratory Syndrome Coronavirus 2 (SARS-CoV-2) has led to the ongoing and relentless global Coronavirus Disease 2019 (COVID19) pandemic, and was the leading cause of death in the 
United States in 2020 (US) [1, 2]. Underlying cardiovascular disease (CVD) risk factors and CVD are important prognosticators of clinical outcomes in hospitalized patients with COVID-19 [3]. Patients with COVID-19 who have pre-existing malignancy and those undergoing cancer-specific therapy are disproportionately affected and represent a population at risk for worse outcomes [3-6]. However, data on the impact of COVID-19 in patients with a history of prior or active cancer who also have underlying or acquired CVD from cancer-related treatments-the cardio-oncology population-is limited. Furthermore, it is important to investigate the safety of recent use of cancer therapies as observational studies early in the pandemic suggested that COVID-19 patients with cancer who are immunosuppressed as a result of their underlying active cytoreduction therapies may have increased mortality and intensive care needs compared to those without cancer [7]. Although the direct impact of COVID-19 on cardio-oncology patients has not been evaluated in clinical trials, prior studies have shown that both CVD patients and cancer patients do have an increased risk for cardiac injury, which in of itself is an independent predictor of mortality in COVID-19 [8, 9]. As oncologists and cardio-oncologists weigh the risk and benefits of initiating versus delaying or continuing versus stopping cancer-related therapies in light of the ongoing pandemic, it is critical to understand the impact of COVID-19 in the cancer population, and particularly among those with concomitant CVD.

To address this evidence gap, we evaluated the association of history of CVD, history of cancer, and recent cancer-related therapy with in-hospital death, as well as other adverse cardiovascular, pulmonary, and venous thromboembolic outcomes among adult patients hospitalized with COVID-19 in the multicenter American Heart Association's (AHA) COVID-19 Cardiovascular disease (CVD) Registry powered by Get With The Guidelines (GWTG).

\section{Methods}

\section{Data acquisition and source}

We obtained patient-level data from 86 US hospitals in the GWTG $^{\circledR}$ COVID-19 CVD registry (March through Aug 20, 2020). The $\mathrm{GWTG}^{\circledR}$ programs are provided by the AHA. The registry is a voluntary effort, and enrollment is available to all health systems in the US treating adult patients with active COVID-19. Participating hospitals are instructed to abstract detailed demographic and clinical data from the medical record of consecutive patients for the duration of the hospital stay. Data are entered into an electronic interactive case record form, which has error checks embedded within the software platform to ensure data quality and prevent erroneous data entry by trained clinical personnel. As the registry is designed as a quality improvement tool, with no intervention or participant contact, informed consent was not obtained and institutional review board exemption was obtained. Detailed methods of the registry have been previously described [10].

\section{Study population, design, and definitions}

The study cohort for the present analyses included all hospitalized adults ( $\geq 18$ years old) with at least one documented admission symptom (Table) associated with confirmed active COVID-19. An active infection was described as having a postivie RT-PCR test either prior to or during hospitalization, a positive IgM antibody test, or a clinical diagnosis using hospital specific criteria. The primary exposure of interest was history of cancer. Patients were determined to have a history of cancer if they had active or prior solid or hematological cancer regardless of active therapy. Key variables of interest included patient's having a history of CVD or recent chemotherapy or biologic therapy (defined as chemotherapy within 2 weeks of hospitalization). CVD was defined as a composite of history of cerebrovascular accident (CVA), history of heart failure (HF), prior myocardial infarction (MI), prior coronary artery bypass graft (CABG), or prior percutaneous coronary intervention (PCI). Other covariates included CVD risk factors [age, gender, BMI, diabetes, hypertension, hyperlipidemia, and history of smoking (cigarette use within 1 year of hospitalization)], pulmonary disease (history of chronic obstructive lung disease, interstitial lung disease, asthma, pulmonary arterial emphysema, chronic bronchitis or being treated for respiratory symptoms with inhaled or oral pharmacological therapy), and chronic kidney disease (physician diagnosis of renal insufficiency, chronic failure, or serum creatinine greater than $2.0 \mathrm{mg} / \mathrm{dl}$ ).

\section{Study endpoints}

The primary outcome was in-hospital death. Four secondary outcomes were evaluated. Secondary outcomes included major adverse cardiovascular events (MACEa composite of in-hospital stroke, heart failure, myocardial infarction, sustained ventricular arrhythmia, or heart block requiring temporary or permanent pacemaker), severe disease complications (composite of new need for hemodialysis, mechanical ventilation, in-hospital shock, inotropic or vasopressor use, or mechanical circulatory support use with use of intra-aortic balloon pump, percutaneous ventricular assist device, veno-arterial or veno-veno extracorporeal membrane oxygenation), or thromboembolic disease (a composite of deep vein thrombosis (DVT) or pulmonary embolism [PE]). 


\section{Statistical analysis}

Data in tables are presented as either mean (standard deviation) or frequency (percentage) for the total study cohort and stratified by cancer history. Continuous and categorical variables were compared with the unpaired $\mathrm{t}$ tests or chi-square tests, respectively. Multivariable logistic regression analyses were performed for the primary and secondary outcomes; data are presented as adjusted odds ratios [aOR] with $95 \%$ confidence intervals $(\mathrm{CI})$. In addition to the main effect term of history of cancer, interaction terms for history of cancer with CVD and CVD risk factors were tested for the primary outcome of in-hospital mortality. Interaction terms that were significant for in-hospital mortality were retained and presented in the final multivariable model. Similar, separate, modeling was done for each of the secondary outcomes. For the primary outcome of in-hospital mortality, a sensitivity analysis was conducted by limiting the cohort to only those with a history of cancer and generating an adjusted model with remaining covariates. All models were adjusted for body mass index (BMI), sex, race, diabetes, dyslipidemia, hypertension, chronic kidney disease, smoking history, and history of pulmonary disease. Generalized estimating equation method was used to estimate the parameters for the multivariable logistic regression models and to account for the correlated structure of the data due to clustering within hospitals. All statistical tests were two-sided, and a $p$-value $<0.05$ was considered statistically significant. All analyses were performed with SAS version 9.4 (Cary, NC) using a deidentified data collected and coordinated via IQVIA (Parsippany, NJ) and housed on the American Heart Association Precision Medicine Platform (precision.heart.org) [10].

\section{Results}

\section{Patient population and baseline characteristics}

Among 86 US hospitals, 8,222 patients through August 20, 2020 were included in the analysis. Among these patients, $892(10.8 \%)$ patients had a history of cancer, $1,805(22.0 \%)$ had a history of CVD, and 261 (3.2\%) had both a history of cancer and CVD. Among cancer patients, 124 (14.0\%) had recent use of chemotherapy (i.e. within 2 weeks of hospitalization). Patients with COVID-19 and a history of cancer were older and had a higher prevalence of CKD, pulmonary disease, and CVD (Table 1). Notably, compared with non-cancer patients, a similar amount of COVID-19 specific novel therapies were administered to cancer patients during their hospitalization.

\section{Association of cancer with in-hospital mortality}

In the entire cohort of adult patients hospitalized with symptomatic COVID-19, 1501 (18.3\%) died during their hospitalization [median occurrence on hospital day 7 (interquartile range [IQR] 3-13 days)], 25.4\% those with cancer and $17.3 \%$ of those without cancer. In univariate analysis, history of cancer was a significant predictor of in-hospital mortality (OR $=1.62,95 \% \mathrm{CI}$ : $1.38-1.91$, $p<0.0001)$. Although no significant interaction between a history of cancer and a history of CVD was found, there were significant interactions between history of cancer with the CVD risk factors of age, BMI and history of smoking for in-hospital mortality. In multivariable analysis, history of cancer was a significant predictor of inhospital mortality (among average age and BMI patients, $\mathrm{aOR}=3.60$, 95\% CI: 2.07-6.24; $p<0.0001$ in those with a smoking history and $\mathrm{aOR}=1.33,95 \% \mathrm{CI}$ : $1.01-1.76$; $p=0.04$ in non-smokers). The largest effect of cancer history on in-hospital mortality was observed in those patients with a history of smoking who were younger and had lower BMIs (Fig. 1). Of note, although history of CVD was associated with in-hospital mortality in univariate analysis $(\mathrm{OR}=2.23,95 \% \mathrm{CI}: 1.98-2.52, p=0.010)$, it did not reach statistical significance for in-hospital mortality in multivariable analysis $(\mathrm{aOR}=1.18,95 \% \mathrm{CI}$ : 0.99 - 1.41, $p=0.07$ ). Additional significant predictors of inhospital mortality included age, male gender, BMI among those without a history of cancer, hypertension, diabetes, chronic kidney disease, pulmonary disease, and smoking among those with a history of cancer.

Among cancer patients, in-hospital mortality occurred in $31.0 \%$ of those with a history of prior CVD as compared to $23.1 \%$ in those without a history of underlying CVD. In adjusted analysis, there was no clear association of CVD with in-hospital mortality $(\mathrm{aOR}=1.01,95 \% \mathrm{CI}$ : $0.63-1.62, p=0.95$ ) (Table 2). In contrast, recent use of chemotherapy was significantly associated with in-hospital mortality among cancer patients $(\mathrm{aOR}=1.72,95 \% \mathrm{CI}$ : $1.05-2.80, p=0.03$ ) (Table 2).

\section{Association of cancer with secondary outcomes}

MACE occurred in 76 (8.6\%) patients with and 397 (5.5\%) without a history of cancer with a median occurrence on day 1 of hospitalization (IQR 0-5 days). Although no significant interaction between history of cancer and history of CVD was found, there were significant interactions between history of cancer and gender. In multivariable analysis, history of cancer was a significant predictor of MACE among females $(\mathrm{aOR}=1.59,95 \% \mathrm{CI}: 1.02-2.48$, $P=0.04$ ), but not males (Table 3 ).

Severe disease complications occurred in 1587 (21.8\%) patients with and 214 (24.2\%) without a history of cancer 
Table 1 Baseline characteristics of symptomatic adult patients hospitalized with COVID-19

\begin{tabular}{|c|c|c|c|c|c|}
\hline \multirow[b]{2}{*}{ Characteristic } & \multicolumn{5}{|c|}{ History of Cancer } \\
\hline & \multicolumn{2}{|l|}{ No $(N=7330)$} & \multicolumn{2}{|l|}{ Yes $(N=892)$} & $\begin{array}{l}p \\
\text { value }\end{array}$ \\
\hline Age (years) & $60.4(17.4)$ & & $70.6(14.1)$ & & $<0.001$ \\
\hline Female Sex & $3231(44.1)$ & & $421(47.2)$ & & 0.077 \\
\hline $\mathrm{BMI}\left(\mathrm{kg} / \mathrm{m}^{2}\right)$ & $30.6(8.2)$ & & $28.5(7.7)$ & & $<0.001$ \\
\hline \multicolumn{6}{|l|}{ Race } \\
\hline White & $4522(61.7)$ & & & $598(67.0)$ & 0.009 \\
\hline Black & $1736(23.7)$ & & & $194(21.8)$ & \\
\hline Asian/Pacific Islander & $469(6.4)$ & & & $46(5.2)$ & \\
\hline Other & $603(8.2)$ & & & $54(6.1)$ & \\
\hline \multicolumn{6}{|l|}{ Payment Source } \\
\hline Uninsured & $521(7.1)$ & & & $22(2.5)$ & $<0.001$ \\
\hline Private & $3946(54.0)$ & & & $459(51.6)$ & \\
\hline Medicare & $1267(17.4)$ & & & $274(30.7)$ & \\
\hline Medicaid & $1310(17.9)$ & & & $124(13.9)$ & \\
\hline Other & $259(3.6)$ & & & $11(1.2)$ & \\
\hline Atrial Fibrillation/Flutter & $626(8.5)$ & $131(14.7)$ & & & $<0.001$ \\
\hline Diabetes Mellitus & $2722(37.1)$ & $328(36.8)$ & & & 0.832 \\
\hline Pulmonary Disease & $2522(34.4)$ & $380(42.6)$ & & & $<0.001$ \\
\hline Chronic Kidney Disease & $928(12.7)$ & $146(16.4)$ & & & 0.002 \\
\hline Hypertension & $4287(58.5)$ & $616(69.1)$ & & & $<0.001$ \\
\hline Smoking History & $461(6.3)$ & $61(6.8)$ & & & 0.53 \\
\hline Pulmonary Disease & $1305(17.8)$ & $203(22.8)$ & & & $<0.001$ \\
\hline \multicolumn{6}{|l|}{ Admission Symptoms } \\
\hline Fever/Chills & $4823(65.8)$ & & & $550(61.7)$ & 0.014 \\
\hline Cough & $4867(66.4)$ & & & $534(59.9)$ & $<0.001$ \\
\hline Shortness of Breath & $4552(62.1)$ & & & $513(57.5)$ & 0.008 \\
\hline Fatigue & $1845(25.2)$ & & & $232(26.0)$ & 0.59 \\
\hline Headache & $722(9.9)$ & & & $49(5.49)$ & $<0.001$ \\
\hline Myalgia & $1580(21.6)$ & & & $188(21.1)$ & 0.74 \\
\hline Sore Throat & $506(6.9)$ & & & $53(5.94)$ & 0.28 \\
\hline Nasal Congestion & $375(5.1)$ & & & $53(5.94)$ & 0.30 \\
\hline Nausea/Nomiting/ Diarrhea & $2066(28.2)$ & & & $254(28.5)$ & 0.86 \\
\hline Loss of Smell/Taste & $303(4.1)$ & & & $44(4.9)$ & 0.26 \\
\hline Confusion or AMS & $770(10.5)$ & & & $145(16.3)$ & $<0.001$ \\
\hline \multicolumn{6}{|l|}{ COVID-19 Therapies } \\
\hline Corticosteroid & $1547(21.8)$ & & & $203(24.7)$ & 0.056 \\
\hline Immunoglobulin & $53(0.7)$ & & & $12(1.4)$ & 0.05 \\
\hline Convalescent serum & $195(2.7)$ & & & $27(3.1)$ & 0.54 \\
\hline Ritonavir/Lopinavir & $76(1.1)$ & & & $7(0.9)$ & 0.60 \\
\hline Remdesivir & $565(7.8)$ & & & $105(11.9)$ & $<0.001$ \\
\hline Tocilizumab & $523(7.2)$ & & & $62(7.0)$ & 0.82 \\
\hline Chemotherapy/biologics & $14(0.19)$ & & & $124(14.0)$ & $<0.001$ \\
\hline History of CVD & $1544(21.1)$ & & & $261(29.3)$ & $<0.001$ \\
\hline
\end{tabular}

Values are depicted as mean (standard deviation) or percentage $(\mathrm{N})$ when appropriate $B M I$ Body Mass Index, AMS Altered Mental Status, CVD Cardiovascular Disease 


Fig. 1 In-Hospital Mortality in Symptomatic COVID-19 Patients by Cancer Status. In multivariable analysis, age, BMI, and history of smoking
were found to have significant interactions with history of cancer for the outcome of in-hospital mortality. AORs for in-hospital mortality among
those with a history of cancer compared to those without a history of cancer are depicted at different values of age and BMl in smokers (A) and
non-smokers (B). The largest effect of cancer history on mortality (darkest red aOR tier) is seen among those with a history of smoking who are
younger and have lower BMls with diminishing effects as individuals become older and more obese (blue aOR tier). aOR, adjusted Odds Ratio; BMI,
Body Mass Index; CVD, Cardiovascular Disease

Table 2 Predictors of in-hospital all-cause mortality in cancer patients admitted with symptomatic COVID-19

\begin{tabular}{|c|c|c|c|}
\hline & & $\begin{array}{l}\text { Multivariable } \\
\text { Analysis }\end{array}$ & $p$ value \\
\hline & OR & $95 \% \mathrm{Cl}$ & \\
\hline Age (per 10 years) & 1.43 & $1.22-1.67$ & $<0.0001$ \\
\hline Female Gender & 0.59 & $0.44-0.79$ & 0.001 \\
\hline BMI (per 10 units) & 0.99 & $0.78-1.25$ & 0.92 \\
\hline Black Race ${ }^{a}$ & 1.15 & $0.80-1.65$ & 0.46 \\
\hline Asian or Pacific Islander Race ${ }^{a}$ & 1.43 & $0.80-2.53$ & 0.22 \\
\hline Undetermined or Other Race ${ }^{a}$ & 1.05 & $0.66-1.70$ & 0.83 \\
\hline Diabetes & 1.13 & $0.82-1.57$ & 0.45 \\
\hline Hypertension & 1.34 & $0.88-2.04$ & 0.17 \\
\hline Hyperlipidemia & 1.12 & $0.81-1.53$ & 0.50 \\
\hline Smoking History & 2.63 & $1.54-4.48$ & $<0.0001$ \\
\hline CKD & 0.85 & $0.60-1.20$ & 0.35 \\
\hline Pulmonary Disease & 1.47 & $1.03-2.09$ & 0.033 \\
\hline CVD History & 1.01 & $0.63-1.62$ & 0.95 \\
\hline Chemotherapy or Biologic Therapy & 1.72 & $1.05-2.80$ & 0.030 \\
\hline
\end{tabular}

BMI Body Mass Index, CVD Cardiovascular Disease, CI Confidence Interval, CKD Chronic Kidney Disease, OR Odds Ratio

${ }^{\text {a } A s ~ c o m p a r e d ~ t o ~ C a u c a s i a n ~ w h i t e ~ r a c e ~}$

with a median occurrence on day 1 of hospitalization (IQR 0-3 days). Although no significant interaction between history of cancer and history of CVD was found for severe disease complications, there were significant interactions between history of cancer with the CVD risk factors of age and smoking history. In multivariable analysis, a history of cancer was a significant predictor of severe disease among smokers $(\mathrm{aOR}=2.76,95 \% \mathrm{CI}$ : 1.98-3.86; $p<0.001$ ) (Table 3).

Thromboembolic disease occurred in 29 (3.3\%) patients with and 198 (2.7\%) without a history of cancer with a median occurrence on day 6 of hospitalization (interquartile range, 1-12 days). History of cancer was not associated with thromboembolic disease in univariable or multivariable analysis (Table 3 ).

\section{Discussion}

This large multicenter AHA COVID-19 CVD registry-based analysis highlights several important points regarding hospitalized adult cancer patients with symptomatic COVID-19. First, a history of cancer was found to be a significant predictor of in-hospital mortality, with its largest effect being in patients who were younger with lower BMIs and had a history of smoking. The higher rates of mortality seem to be driven by severe disease complications. Second, cancer patients who received recent chemotherapy in the prior 2 weeks to admission had increased in-hospital mortality compared with those without recent cancer-related therapy. Finally, cancer patients with concomitant pre-existing CVD had similar rates of in-hospital mortality to those cancer patients 
Table 3 Cardiovascular disease and cancer history association with major complications in patients with symptomatic COVID-19 admissions

\begin{tabular}{|c|c|c|c|c|c|c|}
\hline \multirow[t]{2}{*}{ Outcome of interest } & \multicolumn{3}{|c|}{ Univariable Analysis } & \multicolumn{3}{|c|}{ Multivariable analysis } \\
\hline & OR & $95 \% \mathrm{Cl}$ & $p$ value & OR & $95 \% \mathrm{Cl}$ & $p$ value \\
\hline \multicolumn{7}{|l|}{ Mace } \\
\hline CVD History & 2.06 & $1.69-2.50$ & $<0.001$ & 1.32 & $1.05-1.67$ & 0.018 \\
\hline Cancer History $^{\mathrm{a}}$ & 1.63 & $1.26-2.11$ & $<0.001$ & & & \\
\hline Among Males & & & & 0.85 & $0.65-1.12$ & 0.26 \\
\hline Among Females & & & & 1.59 & $1.02-2.48$ & 0.039 \\
\hline \multicolumn{7}{|c|}{ Severe Disease Complication } \\
\hline CVD History & 1.26 & $1.12-1.41$ & $<0.0001$ & 0.91 & $0.78-1.07$ & 0.26 \\
\hline Cancer History ${ }^{b}$ & 1.15 & $0.99-1.34$ & 0.062 & & & \\
\hline Among Smokers ${ }^{c}$ & & & & 2.76 & $1.98-3.86$ & $<0.001$ \\
\hline Among Non-Smoker ${ }^{c}$ & & & & 1.12 & $0.91-1.37$ & 0.29 \\
\hline \multicolumn{7}{|l|}{ Thromboembolic Event } \\
\hline CVD History & 1.01 & $0.73-1.38$ & 0.97 & 0.93 & $0.68-1.28$ & 0.653 \\
\hline Cancer History & 1.21 & $0.82-1.80$ & 0.34 & 0.86 & $0.55-1.32$ & 0.482 \\
\hline
\end{tabular}

All multivariable models were adjusted for age, gender, body mass index, race, diabetes, hypertension, hyperlipidemia, smoking history, chronic kidney disease, and pulmonary disease

CVD Cardiovascular Disease

${ }^{a}$ The following interaction term is found to be significant in the multivariable logistic regression analysis for the outcome of MACE and retained in the final model: Gender*Cancer History $(p=0.03)$

b The following interaction terms are found to be significant in the multivariable logistic regression analysis for the outcome of severe disease complications and retained in the final model: Age ${ }^{*}$ Cancer History $(p=0.01)$ and Smoking History*Cancer History $(p=0.003)$

$c$ Evaluated at an average age (61.5 years) in the study cohort

without CVD. However, CVD risk factors of age, male gender, BMI, hypertension, diabetes, and smoking were significant predictors in adjusted models.

In 2020, COVID-19, heart disease, and malignant neoplasms have been the leading causes of death in the US [2]. As COVID-19 rates have exponentially increased since March 2020, the interplay between these 3 entities has become increasingly important. Small studies from China at the onset of the pandemic indicated that higher proportions of patients with a history of cancer had severe in-hospital complications and in-hospital mortality as compared to those without a history of cancer, prompting suggestions that intentional postponement of adjuvant chemotherapy or elective surgery should be considered $[7,11]$. With respect to cancer therapies, a small prospective cohort study reported no significant association between use of cytotoxic chemotherapy or other anticancer treatment and mortality in the setting of COVID-19 [12]. A larger cohort study from the COVID-19 and Cancer Consortium similarly reported that cancer patients receiving anticancer therapies did not have higher rates of mortality, although the presence of cancer itself, whether stable/improving or progressive was independently associated with 30-day mortality [13]. However, specific CVD risk factors such as hypertension and obesity, previously shown to be associated with in-hospital mortality were not adjusted for $[14,15]$. More recently, as the pandemic has continued to unfold, there has been more interest in evaluating risk in those with concomitant CVD and history of cancer. For example, in a retrospective study by Ganatra et al. of 2,476 patients from 4 Massachusetts-based hospitals patients with a history of both CVD $(N=414)$ and cancer $(N=195)$ had a higher short-term mortality as compared to either cancer or CVD alone [16]. In addition, history of cancer was predictive of severe disease complications, while preexisting CVD was not.

In the present study, history of cancer was a robust predictor of in-hospital mortality among hospitalized symptomatic adult COVID-19 patients. Given older age and obesity have previously been shown to be associated with mortality in the setting of COVID-19, it is not surprising that the most predominant effect of history of cancer on mortality we observed was in younger patients with lower BMIs. The increased mortality appears to be driven by increased severe disease complications in patients with history of cancer, particularly in those with a history of smoking, independent of prior pulmonary disease. Additionally, while male gender was unsurprisingly associated with higher MACE in those without cancer history, females with a history of cancer exhibited higher rates of MACE. However, interpretation is limited 
for MACE given the overall low event rate. Other factors that may also explain the increased mortality rate observed in cancer patients compared with non-cancer patients include: 1) residual confounding due to unaccounted for differences between cancer and non-cancer patients, such as use of vasoactive medications and code status; and 2) inadequate power to detect a difference in venous thromboembolism (VTE) given low event rates.

Venous thromboembolism (VTE) has been of particular interest in COVID-19 patients with underlying cancer given the perceived elevated risk of VTE from both the hypercoagulable/inflammatory milieu of COVID-19 and cancer itself. Data has shown that elevated D-dimers levels are independently associated with thrombotic events, and subsequently those with thrombotic events have higher all-cause mortality [17]. However, the prevalence of VTE in COVID-19 cancer patients is not clear. Ganatra et al. reported VTE in 10\% of cancer patients admitted with COVID-19, whereas we observed VTE in only $3 \%$ of cancer patients with COVID-19 [16]. This may be an underestimation given other smaller studies suggest higher rates even in those without a history of cancer [18], and may be a reflection of low rates of systematic surveillance and suspicion without confirmation in critically ill patients.

To the best of our knowledge, this AHA COVID-19 registry-based study is the largest multicenter prospective analysis to date to evaluate the effect of recent use of cancer-related therapy in hospitalized symptomatic COVID-19 patients. In contrast to prior studies, among cancer patients, we observed a strong independent association of cancer-specific therapy with in-hospital mortality. Importantly, cancer patients with underlying CVD did not have higher in-hospital mortality rates compared with those without CVD. Taken together, these findings suggest that oncologists and cardio-oncologists alike should discuss with their patients the optimal timing for and possible delay of cancer-related therapy in areas where COVID-19 viral transmission is high and hospital resources are limited, but that the presence of CVD should not be a major determinant of therapy in the cardio-oncology population. However, this be balanced with the need of urgent therapy based on cancer type and cancer status. Multiple societal documents have similarly discussed the need to potentially curtailing the frequency of cardiotoxicity surveillance for selected patients [19-21].

This study has important limitations. First, the AHA CVD COVID-19 registry does not provide granular data regarding the type of cancer, status of cancer (relapse, remission, etc.), or cancer-specific therapies that patients are receiving to allow for stratification of cancer severity. Second, the initial registry data did not include information regarding code status and advanced directives, which may be disproportionately high in those with cancer and thus possibly impact in-hospital outcomes. Third, these data only pertain to in-hospital outcomes and thus post-discharge outcomes are not known. Lastly, the voluntary hospital participation in the registry is weighted toward urban academically affiliated institutions, and so the results may not be generalizable; however, we adjusted for site-specific clustering of outcomes to address this issue.

\section{Conclusion}

In summary, in the large national AHA COVID-19 CVD registry, history of cancer is a significant predictor of inhospital mortality. Notably, among cancer patients, those with underlying CVD had similar outcomes to those without CVD, while recent use of cancer-related therapy was significantly associated with in hospital mortality. Further studies focusing on malignancy type and specific treatments, and their interactions with concomitant CVD and CVD risk factors, are warranted to identify vulnerable subgroups of the cancer population that may benefit from aggressive interventions during the COVID19 pandemic including earlier prioritization for vaccine initiation.

\section{Acknowledgements}

The Get With The Guidelines ${ }^{\circledR}$ programs are provided by the American Heart Association. The American Heart Association Precision Medicine Platform (nhttps://precision.heart.org/) was used for data analysis. IQVIA (Parsippany, New Jersey) serves as the data collection and coordination center. Statistical support has been provided via the UCLA Clinical and Translational Science Institute (grant number UTR001881).

\section{Authors' contributions}

All authors read and approved the final manuscript. All authors made substantial contributions to the conception and design of the work. DMT and $\mathrm{XW}$ analyzed the data. DMT, AMR, RVP, and EHY contributed to drafting the manuscript.

\section{Funding}

Statistical support has been provided via the UCLA Clinical and Translational Science Institute (grant number UTR001881). AHA's suite of Registries is funded by multiple industry sponsors. AHA's COVID-19 CVD Registry is partially supported by The Gordon and Betty Moore Foundation.

\section{Availability of data and materials}

The data that support the findings of this study are available from AHA COVID19 Registry but restrictions apply to the availability of these data, which were used under license for the current study, and so are not publicly available. Data are however available from the authors upon reasonable request and with permission of AHA COVID-19 Registry.

\section{Declarations}

Ethics Approval and Consent to participate

Ethical approval not applicable. Informed consent was waived in the setting of centralized de-identified data.

Consent for publication

Not applicable. 


\section{Competing interests}

$\mathrm{JH}$ reports unrelated grant support from the $\mathrm{NIH} / \mathrm{NCI}$ (RO1-CA 233610). TGH reports unrelated activities including advisory fees from Parexel, BMS, H3 Biomedicine, AbbVie, and Intrinsic Imaging and grant support fufrom Astra Zeneca, the Kohlberg Foundation, the NIH/NHLBI (RO1HL130539, RO1HL137562, and K24HL150238), and the NIH/Harvard Center for AIDS Research (P30 Al060354). RVP reports unrelated activities including research support from the American Heart Association and Janssen, and serving on the scientific advisory board (minor equity interest) of Stallion Cardio and HeartCloud. EHY reports unrelated activities including research support from CSL Behring.

\section{Author details}

'Division of Cardiology, Department of Medicine, University of California, Los Angeles Health System, Los Angeles, CA, USA. ${ }^{2}$ Division of General Internal Medicine and Health Service Research, Department of Medicine, University of California, Los Angeles Health System, Los Angeles, CA, USA. ${ }^{3}$ Division of Cardiology, Department of Medicine, Michigan Medicine, Ann Arbor, MI, USA. ${ }^{4}$ Department of Cardiovascular Diseases, Mayo Clinic, Rochester, MN, USA. ${ }^{5}$ Division of Cardiology, Department of Medicine, Massachusetts General Hospital, Boston, MA, USA. ${ }^{6}$ Department of Medicine, University of California, Los Angeles Health System, Los Angeles, CA, USA. ${ }^{7}$ Division of Hematology and Oncology, Department of Medicine, Northwestern Memorial Hospital, Chicago, IL, USA. ${ }^{8}$ Division of Cardiology, Department of Medicine, Texas MD Anderson Cancer Center, Houston, TX, USA. ' UCLA Cardio-Oncology Program, Division of Cardiology, Department of Medicine, University of California, Los Angeles Health System, Los Angeles, CA, USA

\section{Received: 4 June 2021 Accepted: 14 July 2021}

Published online: 10 August 2021

\section{References}

1. COVID-19 Dashboard by the Center for Systems Science and Engineering at Johns Hopkins University. https://gisanddata.maps.arcgis.com/apps/ opsdashboard/index.html\#/bda7594740fd40299423467b48e9ecf6. Last Accessed 20 Sept 2020.

2. Woolf SH, Chapman DA, Lee JH. COVID-19 as the leading cause of death in the United states. JAMA. 2020. https://doi.org/10.1001/jama.2020 24865.

3. Richardson S, Hirsch JS, Narasimhan M, et al. Presenting characteristics, comorbidities, and outcomes among 5700 patients hospitalized with COVID-19 in the New York City area. JAMA. 2020;323(20):2052-9.

4. Goyal P, Choi JJ, Pinheiro LC, Schenck EJ, Chen R, Jabri A, Satlin MJ Campion TR Jr, Nahid M, Ringel JB, Hoffman KL. Clinical characteristics of Covid-19 in New York City. N Engl J Med. 2020;382(24):2372-4.

5. Wu Z, McGoogan JM. Characteristics of and important lessons from the coronavirus disease 2019 (COVID-19) outbreak in China: summary of a report of 72314 cases from the Chinese center for disease control and prevention. JAMA. 2020;323(13):1239-42.

6. Onder G, Rezza G, Brusaferro S. Case-fatality rate and characteristics of patients dying in relation to COVID-19 in Italy. JAMA. 2020;323(18):1775-6.

7. Liang W, Guan W, Chen R, Wang W, Li J, Xu K, Li C, Ai Q, Lu W, Liang H, Li S. Cancer patients in SARS-CoV-2 infection: a nationwide analysis in China. Lancet Oncol. 2020;21(3):335-7.
8. Shi S, Qin M, Shen B, et al. Association of cardiac injury with mortality in hospitalized patients with COVID-19 in Wuhan. China JAMA Cardiology. 2020:5(7):802-10.

9. Januzzi JL, Johnson KW, Lala A, et al. prevalence of myocardial injury in patients hospitalized with COVID-19 infection. J Am Coll Cardiol. 2020;76(5):533-46.

10. Alger HM, Rutan C, Williams JH, et al. American heart association covid-19 cvd registry powered by get with the guidelines. Circ Cardiovasc Qual Outcomes. 2020;13(8):e006967.

11. Meng Y, Lu W, Guo E, et al. Cancer history is an independent risk factor for mortality in hospitalized COVID-19 patients: a propensity score-matched analysis. J Hematol Oncol. 2020;13(1):75.

12. Lee LY, Cazier JB, Angelis V, et al. COVID-19 Motrality in Patients with Cancer on Chemotherapy or Other Anticancer Treatments: A Prospective Cohort Study. Lancet. 2020;395(10241):1919-26.

13. Kuderer KM, Choueiri TK, Shah DP, et al. Clinical Impact of COVID19 on Patients with Cancer (CCC19): a cohort study. Lancet. 2020;395(10241):1907-18.

14. Cunningham JW, Vaduganathan M, Claggett BL, et al. Clinical Outcomes in Young US Adults Hospitalized With COVID-19. JAMA Intern Med. 2020:e205313. https://doi.org/10.1001/jamainternmed.2020.5313.

15. Hendren NS, de Lemos JA, Ayers C, et al. Association of body mass index and age with morbidity and mortality in patients hospitalized with COVID-19: results from the american heart association covid-19 cardiovascular disease registry. Circulation. 2020. https://doi.org/10.1161/CIRCU LATIONAHA.120.051936.

16. Ganatra S, Dani SS, Redd R, et al. Outcomes of COVID-19 in Patients With a History of Cancer and Comorbid Cardiovascular Disease. J Natl Compr Canc Netw. 2020:1-10. https://doi.org/10.6004/jnccn.2020.7658.

17. Bilaloglu S, Aphinyanaphongs Y, Jones S, et al. Thrombosis in hospitalized patients with COVID-19 in a New York City health system. JAMA. 2020;324(8):799-801. https://doi.org/10.1001/jama.2020.13372.

18. Zhang $L$, Feng $X$, Zhang $D$, et al. Deep vein thrombosis in hospitalized patients with COVID-19 in Wuhan, China: prevalence, risk factors, and outcome. Circulation. 2020;142(2):114-28.

19. Baldassarre LA, Yang EH, Cheng RK, et al. Cardiovascular care of the oncology patient during COVID-19: An expert consensus document from the ACC Cardio-Oncology and Imaging Councils. J Natl Cancer Inst. 2020. https://doi.org/10.1093/jnci/djaa177.

20. Lenihan D, Carver J, Porter C, et al. Cardio-oncology care in the era of the coronavirus disease 2019 (COVID-19) pandemic: An International CardioOncology Society (ICOS) statement. CA Cancer J Clin. 2020;70(6):480-504.

21. Kirkpatrick JN, Mitchell C, Taub C, et al. ASE statement on protection of patients and echocardiography service providers during the 2019 novel coronavirus outbreak: endorsed by the american college of cardiology. J Am Soc Echocardiogr. 2020;33(6):648-53.

\section{Publisher's Note}

Springer Nature remains neutral with regard to jurisdictional claims in published maps and institutional affiliations.

Ready to submit your research? Choose BMC and benefit from

- fast, convenient online submission

- thorough peer review by experienced researchers in your field

- rapid publication on acceptance

- support for research data, including large and complex data types

- gold Open Access which fosters wider collaboration and increased citations

- maximum visibility for your research: over 100M website views per year

At $\mathrm{BMC}$, research is always in progress.

Learn more biomedcentral.com/submissions 